\title{
Unregulated Hand Dug Wells and Their Quality Threat: A Case Study in Cape Coast Metropolis
}

\author{
Albert Ebo Duncan*, Michael Oteng Peprah, Martha Osei Marfo \\ Department of Water and Sanitation, School of Physical Science, University of Cape Coast, Cape Coast, Ghana \\ Email: *aduncan@ucc.edu.gh
}

How to cite this paper: Duncan, A.E., Peprah, M.O. and Marfo, M.O. (2020) Unregulated Hand Dug Wells and Their Quality Threat: A Case Study in Cape Coast Metropolis. Open Access Library Journal, 7: e6482.

https://doi.org/10.4236/oalib.1106482

Received: May 29, 2020

Accepted: July 11, 2020

Published: July 14, 2020

Copyright $\odot 2020$ by author(s) and Open Access Library Inc.

This work is licensed under the Creative Commons Attribution International License (CC BY 4.0).

http://creativecommons.org/licenses/by/4.0/ (c) (i) Open Access

\begin{abstract}
In Ghana, using hand-dug wells whose quality has not been scientifically assessed is a common practice. All the hand-dug wells in Abura and its environs where this study took place have not been scientifically assessed; however, they are being used for domestic activities including drinking. In all, 128 water samples were collected from 20 hand-dug wells in the Abura community. Sixteen physical, chemical, and microbial parameters namely $\mathrm{pH}$, turbidity, total suspended solids, conductivity, total dissolved solids, color total hardness, alkalinity, magnesium, calcium, iron, manganese, potassium, fluoride, phosphate, and E. coli were assessed in this study. Microbially, all the wells were found to be unsafe for drinking. The parameters total hardness, manganese, fluoride, and, phosphate were found to be within the permissible threshold for all the wells. In all the wells, the total dissolved solids (TDS) measured were above the threshold. Eleven out of the 20 wells recorded $\mathrm{pH}$ outside the permissible threshold. Eight of the wells recorded color above the permissible threshold. The wells A, D, E, F, G, H, and D, G, I, J, N, O recorded alkalinity and iron concentration outside the permissible threshold. Apart from wells $\mathrm{N}$ and $\mathrm{O}$, all the remaining 18 wells recorded turbidity within the permissible threshold. Only wells $\mathrm{D}, \mathrm{H}$, and $\mathrm{N}$ recorded potassium concentrations within the threshold. The water quality index computations classify the wells E, K, M, P, R, and S as excellent; A, B, C, F, H, L, Q, and T as good; J and $\mathrm{O}$ as poor and very poor; and N, I, G, and D as undesirable for drinking. Of all the parameters analyzed, only TDS polluted all the wells. All the wells were polluted with one type of parameter or another which calls for some level of treatment before use.
\end{abstract}

\section{Subject Areas}

Hydrology

\section{Keywords}

Perception, Groundwater, Safety, Drinking, Cape Coast 


\section{Introduction}

If anyone could survive without drinking from a safe source of water, then it must be for a short period. Globally, about $17 \%$ of the world population doesn't have access to a safe water source [1]. Access to safe water does not only help in the survival of human lives but also provides a good platform for economic growth, industrialization, and environmental flow. Among the raw materials in the world, groundwater is the most extracted and this is estimated at 982 $\mathrm{km}^{3} /$ year [2]. For countries like Malta and Denmark, groundwater is the only source of water supply whereas, in countries like Belgium, Netherlands, and Tunisia groundwater forms $85 \%, 75 \%$, and $95 \%$ of their water resources respectively [3]. Globally, about 38\% of irrigation is dependent on groundwater [4]. About $50 \%$ of drinking water used globally is derived from groundwater sources [5]. There are enormous benefits from the use of groundwater in both urban and rural environments. It is a source of water supply for human survival and economic development in extensive drought-prone areas of south-eastern, eastern and western Africa, especially where the average rainfall is less than 1000 $\mathrm{mm} /$ annum [6]. Even though, there are no comprehensive statistics of urban water supply derived from groundwater [6] no one could dispute the enormous contribution of groundwater for domestic and industrial activities in urban centers especially in developing or third world countries. One reason that could contribute to the increase in the use of groundwater according to [4] is its good quality and stable composition (not prone to seasonal variations) over a long period as compared to surface water. Besides, it is not prone to pollution and microbial contamination, and much more uniformly spread over large regions than surface water. Furthermore, it is usually available in areas where surface water is absent. Finally, its development could be gradual depending on demand contrary to surface water that requires huge capital one-time investment. There is a strong connection between surface and groundwater for environmental sustainability. The flow of groundwater into rivers as seepage through the river bed is not only essential to the health of wildlife and aquatic plants but it also keeps rivers from drying up during dry season [7]. Groundwater irrespective of its rock bearing formation could be contaminated either by natural or anthropogenic means. Natural sources of contamination are strongly influenced by the geological formation, which is the mineral composition and soil-rock-water interaction [8]. Some anthropogenic activities such as the release of untreated or improperly treated waste into the environment could serve as a pollution source as some groundwaters are recharged by surface waters [9] [10]. In countries such as Russia and Bulgaria, strict rules and water legislation are guiding the use of fresh groundwater for domestic and drinking water supply. For instance, fresh groundwater for irrigation and industrial purpose is allowed only when it is sufficient to meet drinking water demands [4]. However, in most developing countries groundwater abstraction is done under no regulation.

In Africa and Ghana specifically, the unregulated abstraction is of great con- 
cern because most groundwater sources along the coastal areas lie close to the surface making them prone to pollution (Figure 1). Compounding the problem in Ghana is the issue of high population growth and intermittent drinking water supply which compels most houses to construct wells and boreholes for water abstraction without following any legislative guidelines or rules. Besides, most of these wells are hand dug, shallow, and are not subjected to any quality testing before use.

This situation is more intense in areas such as the Cape Coast in the Central Region of Ghana. Cape Coast and its environs started facing perennial water shortage since 1987 and no one thought it would escalate to this level in three decades. The crucial water demands some years back due to drought effects across the region and in many parts of the Region and more specifically Cape Coast led to the construction of hand dug-wells in many homes in Abura, Pedu, Eyifua, etc. Unfortunately, the construction of these wells didn't follow any legislative guidelines or rules. Furthermore, most of the wells are shallow, poorly constructed without even lining of the walls and protective structure to prevent runoff inflow. These gaps notwithstanding, the households use the water sources for domestic activities including drinking without any quality check to ascertain the safety of the water. What makes this situation worrying is that newly developing areas also follow suit. This study looks at the threat pose by the unregulated hand-dug wells on the quality of water and the potential threat it poses to the users.

\section{Study Area}

Cape Coast Metropolitan Assembly is one of the 260 Metropolitan, Municipal, and District Assemblies (MMDAs) in Ghana and forms part of the 22 MMDAs in the Central Region of Ghana. The Metropolis covers an area of 122 square kilometers and is the smallest metropolis in the country. It is located on longitude $1^{\circ} 15^{\prime} \mathrm{W}$ and latitude $5^{\circ} 06^{\prime} \mathrm{N}$. The metropolis is part of the Birimian geological formations which is rich in granitoid and shows extensive granitization (Figure 2).

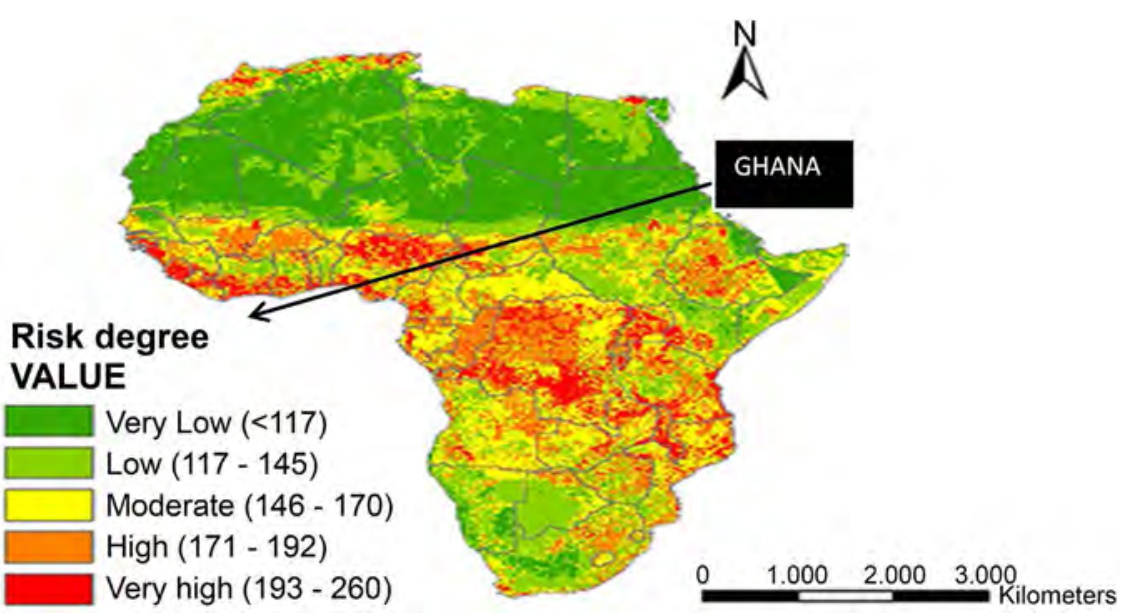

Figure 1. Risk of groundwater pollution across Africa by Paula Park. 

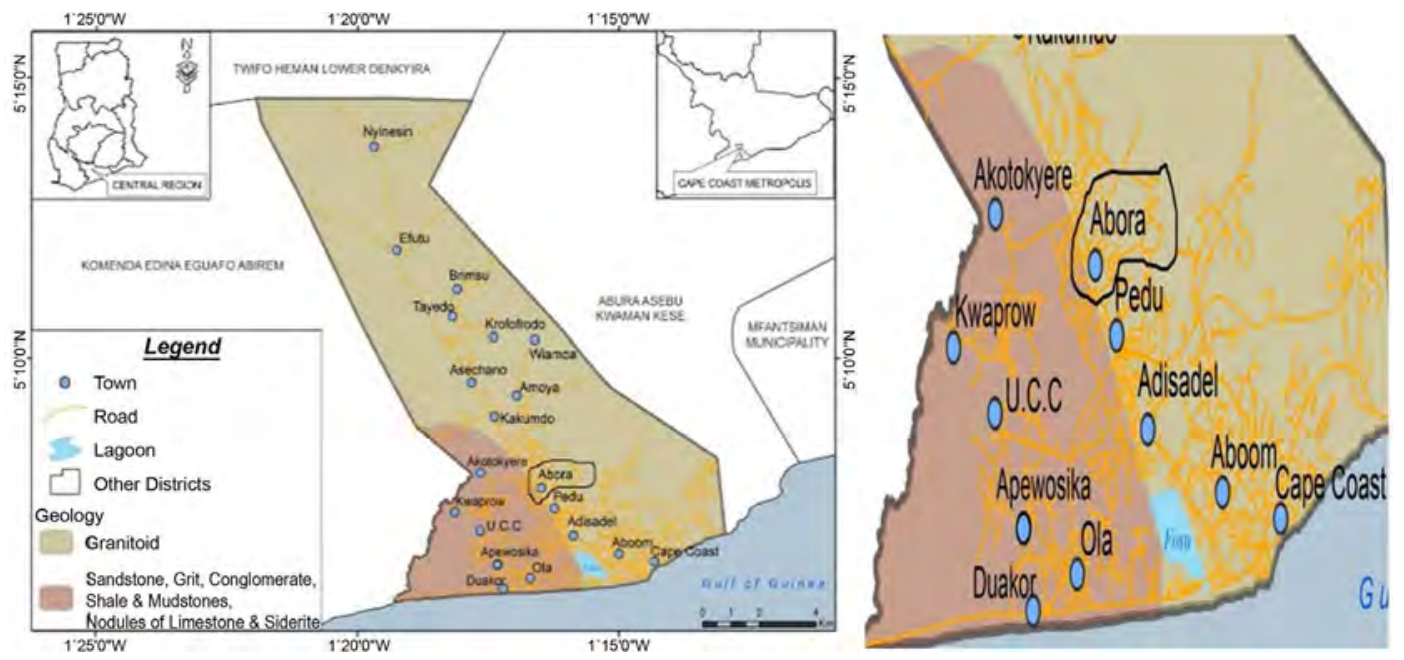

Figure 2. Geological map of Cape Coast.

The administrative capital of the Metropolis is Cape Coast, the first capital town of Ghana. The Cape Coast Metropolitan Area is one of the oldest districts in Ghana. It was raised to the status of a municipality in 1987 by LI 1373 and upgrade to metropolitan status in 2007 by LI 1927. The Metropolis is bounded on the south by the Gulf of Guinea, west by Komenda Edina Eguafo/Abrem Municipal, east by the Abura Asebu Kwamankese District and north by the Twifo Hemang Lower Denkyira District. The population of the Metropolis according to the 2010 population and housing census stands at 169,894 with 82,810 males and 87,084 females. Most of the people are into small scale businesses such as operating supermarkets and hardware stores whereas those living along the coast are mostly fishermen. The focus area of study is Abura where the central regional hospital is located. Abura also shares its boundaries withs institutions like the Cape Coast University. Some of the workers from these institutions live in the Abura community. Due to the intermittent flow of water in Cape Coast and Abura, in particular, most households have resorted to hand-dug wells to augment the low assess to potable water. Unfortunately, most of these wells apart from being very shallow and not well constructed have their quality not empirically measured. Besides, most of these wells are used for domestic activities such as drinking, washing, and cooking. It is therefore prudent to measure the quality of the groundwater to ascertain its quality as well as the health impacts for the designated use.

\section{Sample Collection and Analytical Method}

A total of 128 groundwater samples were collected individually from 20 domestic wells in Abura and its environs (Figure 3) into acid-cleaned high-density 1.5-L linear polyethylene sampling bottles using the sampling protocol as described by [11] and analyzed independently. During sampling, the sampling bottle was rinsed over again with the sample at each point of collection, preserved in ice $\left(4^{\circ} \mathrm{C}\right)$ and transported in ice-cold containers to the laboratory for analysis. 


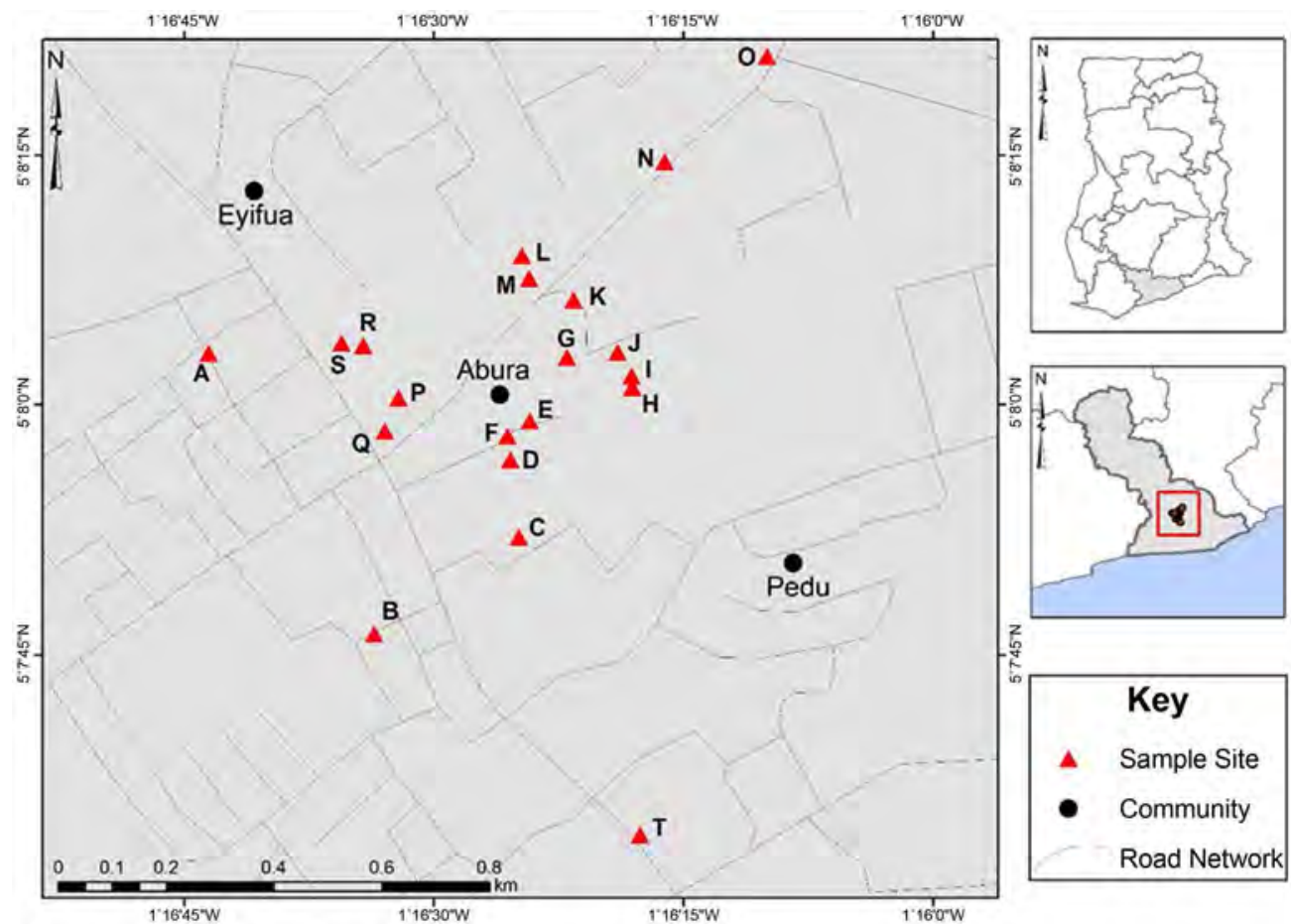

Figure 3. Sampling area.

The $\mathrm{pH}$ was done using a $\mathrm{pH}$ meter (model No. PC320). Electrical conductivity (EC), turbidity, and total dissolved solids (TDS) were measured with the pen-type conductivity meter (model No. LH-P1318), portable 2100Q turbidity meter of the HACH United States of America and portable HACH conductivity meter respectively. Before taking readings, all the equipment was adequately calibrated. Ultrapure metal-free deionized water was used for all analyses. The titrimetric method was employed to determine alkalinity and total hardness. Iron, manganese, fluoride, and phosphate were measured using a spectrophotometer. The flame photometer was used to measure the concentration of potassium.

\subsection{Method of Assessing Water Quality}

In this study, two indices namely the Weighted Arithmetic Water Quality Index (WAWQI) and Nemerow's Pollution Index (NPI) would be applied in the quality assessment. These indices use the permissible levels of the parameters concerned as a reference point for assessment.

\subsubsection{Water Quality Index (WQI)}

A water quality index provides a convenient means of assessing and presenting the overall state or quality of a water source to the general public in simple figures and categorization. Different forms of the index such as the Oregon water quality index (OWQI) (based on the harmonic averaging concept), the National Sanitation Foundation (NSF) in additive WQI and multiplicative WQI, the Canadian Council Ministers of Environment (CCME) WQI, etc. exist, however, the Weighted Arithmetic Index(WAI) (Brown et al., 1972) method was employed in 
this study. The WAIWQI uses a rating scale from 0 to 100 with each range of value having quality implication. A value of zero (0) implies that the water is excellent whereas a value of 100 indicates undesirable drinking water. There are five categories as indicated in Table 1.

The WQI is calculated by the relation:

$$
\mathrm{WQI}=\sum q_{n} W_{n} / \sum W_{n}
$$

The sub-index $q_{n}$ is calculated by the expression:

$$
q_{n}=100 * \frac{V_{n}-V_{i o}}{S_{n}-V_{\text {io }}}
$$

where: $q_{n}=$ Quality rating for the $n^{\text {th }}$ water quality parameter; $V_{n}=$ The estimated parameter for the $n^{\text {th }}$ water quality parameter; $S_{n}=$ Standard value for the $\mathrm{n}^{\text {th }}$ parameter; $V_{i o}=$ ideal value of selected parameters tested (in pure water $V_{i o}$ is 0 ) for all parameters tested. However, for $\mathrm{pH}$ and dissolved oxygen, the value is 7.0 and 14.6 respectively.

$$
W_{n}=\frac{K}{S_{n}}
$$

$S_{n}=$ the standard permissible value for the $n^{\text {th }}$ parameter

$K=$ proportionality constant and is given by the expression

$$
K=\frac{1}{\sum_{n}^{i} \frac{1}{S_{n}}}
$$

In other to assess the polluting potential of the individual parameters, Nemerow's pollution index was used. This index measures the extent of pollution of individual parameters at every sampling location per their standard permissible levels. It is mathematically expressed as:

$$
\mathrm{NPI}=\frac{C_{i}}{L_{i}}
$$

where $C_{i}$ is the observed concentration of the $I^{\text {th }}$ parameter; $L_{i}$ is the permissible limit of the $i^{\text {th }}$ parameter. Each value of the calculated NPI represents the relative pollution contribution by a single parameter. NPI values less than or equal to 1 indicates the absence of pollution and any value above 1 indicates pollution. This means that the higher the measured value, the greater the pollution effect.

Table 1. Water quality index and quality of water.

\begin{tabular}{cc}
\hline Water quality index level & Water quality status \\
\hline $0-25$ & Excellent \\
$26-50$ & Good \\
$51-75$ & Poor \\
$76-100$ & Very poor \\
$100>$ & Undesirable for drinking \\
\hline
\end{tabular}




\section{Results and Discussion}

The results of the physicochemical and biological parameters of the twenty (20) different hand-dug wells measured are presented in Tables 2-9. The results of the physical and chemical parameters indicate that some of the parameters are above their threshold and they are displayed in red color. $\mathrm{pH}$ is an important physicochemical measurement and it indicates the acidity or basicity of water. It is measured using a scale of 0 to 14 . High acidic water has the tendency to corrode metal piping and containers as well as giving a bitter or metallic taste [12]. WHO optimum limits of $\mathrm{pH}$ levels in drinking water are between $6.5-8.5$. The mean $\mathrm{pH}$ range of 5.24 to 6.99 was recorded for all the 20 wells (Table 2). Eleven out of the 20 wells sampled had their $\mathrm{pH}$ outside the WHO standard. The $\mathrm{pH}$ of such wells is acidic and not suitable for drinking purposes.

Turbidity is an expression of the optical property that causes light to be scattered and absorbed somewhat than transmitted with no change in direction or

Table 2. Well water showing mean $\mathrm{pH}$ and turbidity.

\begin{tabular}{|c|c|c|c|c|c|c|}
\hline \multirow{2}{*}{ WELLS } & \multicolumn{3}{|c|}{$\mathrm{pH}$} & \multicolumn{3}{|c|}{ TURBIDITY } \\
\hline & MEAN & MIN & MAX & MEAN (NTU) & MIN & MAX \\
\hline well A & $6.99 \pm 0.08$ & 6.9 & 7.1 & $2.35 \pm 0.15$ & 2.12 & 2.44 \\
\hline well B & $6.36 \pm 0.08$ & 6.32 & 6.38 & $3.55 \pm 0.02$ & 3.52 & 3.57 \\
\hline well C & $6.33 \pm 0.02$ & 6.31 & 6.36 & $1.35 \pm 0.04$ & 1.31 & 1.41 \\
\hline well D & $6.72 \pm 0.02$ & 6.7 & 6.74 & $2.54 \pm 0.01$ & 2.52 & 2.55 \\
\hline well E & $6.86 \pm 0.02$ & 6.84 & 6.88 & $0.65 \pm 0.02$ & 0.63 & 0.67 \\
\hline well F & $6.84 \pm 0.01$ & 6.83 & 6.85 & $0.46 \pm 0.02$ & 0.44 & 0.48 \\
\hline well G & $6.68 \pm 0.02$ & 6.66 & 6.7 & $3.85 \pm 0.06$ & 3.77 & 3.89 \\
\hline well $\mathrm{H}$ & $6.73 \pm 0.02$ & 6.7 & 6.74 & $2.27 \pm 0.06$ & 2.17 & 2.31 \\
\hline well I & $5.47 \pm 0.02$ & 5.44 & 5.49 & $0.68 \pm 0.02$ & 0.66 & 0.71 \\
\hline well J & $6.77 \pm 0.03$ & 6.74 & 6.8 & $1.65 \pm 0.03$ & 1.6 & 1.67 \\
\hline well $\mathrm{K}$ & $5.67 \pm 0.03$ & 5.64 & 5.7 & $1.39 \pm 0.06$ & 1.3 & 1.42 \\
\hline well L & $5.05 \pm 0.02$ & 5.03 & 5.07 & $1.26 \pm 0.01$ & 1.24 & 1.27 \\
\hline well M & $5.26 \pm 0.01$ & 5.24 & 5.27 & $0.32 \pm 0.02$ & 0.3 & 0.34 \\
\hline well $\mathrm{N}$ & $5.50 \pm 0.02$ & 5.48 & 5.52 & $50.70 \pm 0.02$ & 6.5 & 66.7 \\
\hline well O & $6.43 \pm 0.03$ & 6.4 & 6.46 & $9.77 \pm 0.06$ & 9.72 & 9.84 \\
\hline well P & $6.22 \pm 0.05$ & 6.15 & 6.27 & $0.61 \pm 0.03$ & 0.56 & 0.64 \\
\hline well Q & $5.47 \pm 0.01$ & 5.46 & 5.49 & $0.51 \pm 0.01$ & 0.49 & 0.51 \\
\hline well $\mathrm{R}$ & $6.91 \pm 0.01$ & 6.9 & 6.92 & $0.60 \pm 0.03$ & 0.58 & 0.64 \\
\hline well S & $6.63 \pm 0.01$ & 6.61 & 6.64 & $1.75 \pm 0.06$ & 1.67 & 1.8 \\
\hline well T & $5.24 \pm 0.01$ & 5.23 & 5.26 & $1.45 \pm 0.04$ & 1.39 & 1.49 \\
\hline WHO & \multicolumn{3}{|c|}{$6.5-8.5$} & \multicolumn{3}{|c|}{5} \\
\hline
\end{tabular}

Figures highlighted bold are outside the standards. 
Table 3. Well water showing mean TSS and conductivity.

\begin{tabular}{|c|c|c|c|c|c|c|}
\hline \multirow{2}{*}{ WELLS } & \multicolumn{3}{|c|}{ TOTAL SUSPENDED SOLIDS (TSS) } & \multicolumn{3}{|c|}{ CONDUCTIVITY } \\
\hline & MEAN (mg/L) & MIN & MAX & MEAN (mg/L) & MIN & MAX \\
\hline well A & $6 \pm 0.82$ & 5 & 7 & $1552 \pm 6.22$ & 1547 & 1561 \\
\hline well B & $5.25 \pm 1.26$ & 4 & 7 & $1191 \pm 2.45$ & 1188 & 1194 \\
\hline well C & $0 \pm 0.00$ & 0 & 0 & $1177 \pm 4.83$ & 1173 & 1184 \\
\hline well D & $6.5 \pm 0.58$ & 6 & 7 & $1470.25 \pm 3.20$ & 1468 & 1475 \\
\hline well E & $0 \pm 0.00$ & 0 & 0 & $2221.75 \pm 19.55$ & 2210 & 2251 \\
\hline well F & $2.75 \pm 0.96$ & 2 & 4 & $2256.75 \pm 9.60$ & 2250 & 2271 \\
\hline well G & $13.25 \pm 0.96$ & 12 & 14 & $3055.5 \pm 5.97$ & 3050 & 3064 \\
\hline well H & $6 \pm 0.82$ & 5 & 7 & $2127 \pm 9.49$ & 2120 & 2141 \\
\hline well I & $0 \pm 0.00$ & 0 & 0 & $896.5 \pm 1.29$ & 895 & 898 \\
\hline well J & $0.25 \pm 0.50$ & 0 & 1 & $1887 \pm 4.83$ & 1883 & 1894 \\
\hline well $\mathrm{K}$ & $0 \pm 0.00$ & 0 & 0 & $959.75 \pm 1.54$ & 958 & 961 \\
\hline well L & $0.25 \pm 0.50$ & 0 & 1 & $1137 \pm 2.45$ & 1134 & 1139 \\
\hline well M & $1 \pm 0.82$ & 0 & 2 & $933.25 \pm 1.50$ & 931 & 934 \\
\hline well N & $0 \pm 0.00$ & 0 & 0 & $974.75 \pm 1.50$ & 973 & 976 \\
\hline well O & $1 \pm 0.82$ & 0 & 2 & $1430.75 \pm 1.26$ & 1429 & 1432 \\
\hline well P & $1.5 \pm 0.58$ & 1 & 2 & $1376.75 \pm 1.50$ & 1375 & 1378 \\
\hline well Q & $1 \pm 0.82$ & 0 & 2 & $1617.25 \pm 5.85$ & 1611 & 1624 \\
\hline well R & $0.25 \pm 0.50$ & 0 & 1 & $1758.5 \pm 3.87$ & 1755 & 1764 \\
\hline well S & $1.5 \pm 0.58$ & 1 & 2 & $1966.5 \pm 5.07$ & 1963 & 1974 \\
\hline well $\mathrm{T}$ & $0.25 \pm 0.50$ & 0 & 1 & $896.75 \pm 2.99$ & 894 & 901 \\
\hline WHO & & & & \multicolumn{3}{|c|}{1000} \\
\hline
\end{tabular}

Figures highlighted bold are above the standards.

Table 4. Well water showing mean TDS and color.

\begin{tabular}{ccccccc}
\hline \multirow{2}{*}{ WELLS } & \multicolumn{2}{l}{ TOTAL DISSOLVED SOLIDS (TDS) } & \multicolumn{3}{c}{ COLOR } \\
\cline { 2 - 7 } & MEAN (mg/L) & MIN & MAX & MEAN (Pt.Co) & MIN & MAX \\
\hline well A & $1065.25 \pm 4.74$ & 1062.45 & 1072.35 & $39.25 \pm 2.22$ & 37 & 42 \\
well B & $\mathbf{8 0 2 . 7 6 \pm 0 . 8 7}$ & $\mathbf{8 0 1 . 9}$ & 803.6 & $42 \pm 1.83$ & 40 & 44 \\
well C & $792.37 \pm 0.60$ & 791.78 & 793.21 & $8.25 \pm 2.22$ & 6 & 11 \\
well D & $991.80 \pm 0.65$ & 990.9 & 992.4 & $42.5 \pm 2.65$ & 40 & 46 \\
well E & $1493.11 \pm 1.51$ & 1491.75 & 1495.2 & $0 \pm 0.00$ & 0 & 0 \\
well F & $1519.38 \pm 0.68$ & 1518.75 & 1520.35 & $12.5 \pm 1.91$ & 11 & 15 \\
well G & $2058.83 \pm 2.04$ & 2056.2 & 2061.15 & $84 \pm 1.63$ & 82 & 86 \\
well H & $\mathbf{1 4 3 2 . 4 8 \pm 1 . 2 7}$ & 1431 & 1434.1 & $36 \pm 1.63$ & 34 & 38 \\
well I & $\mathbf{6 0 5 . 2 8} \pm 1.03$ & 604.13 & 606.15 & $0 \pm 0.00$ & 0 & 0 \\
well J & $\mathbf{8 9 0 . 4 5 \pm 5 7 2 . 2 6}$ & 127.2 & 1272.1 & $33 \pm 2.45$ & 31 & 36 \\
\hline
\end{tabular}




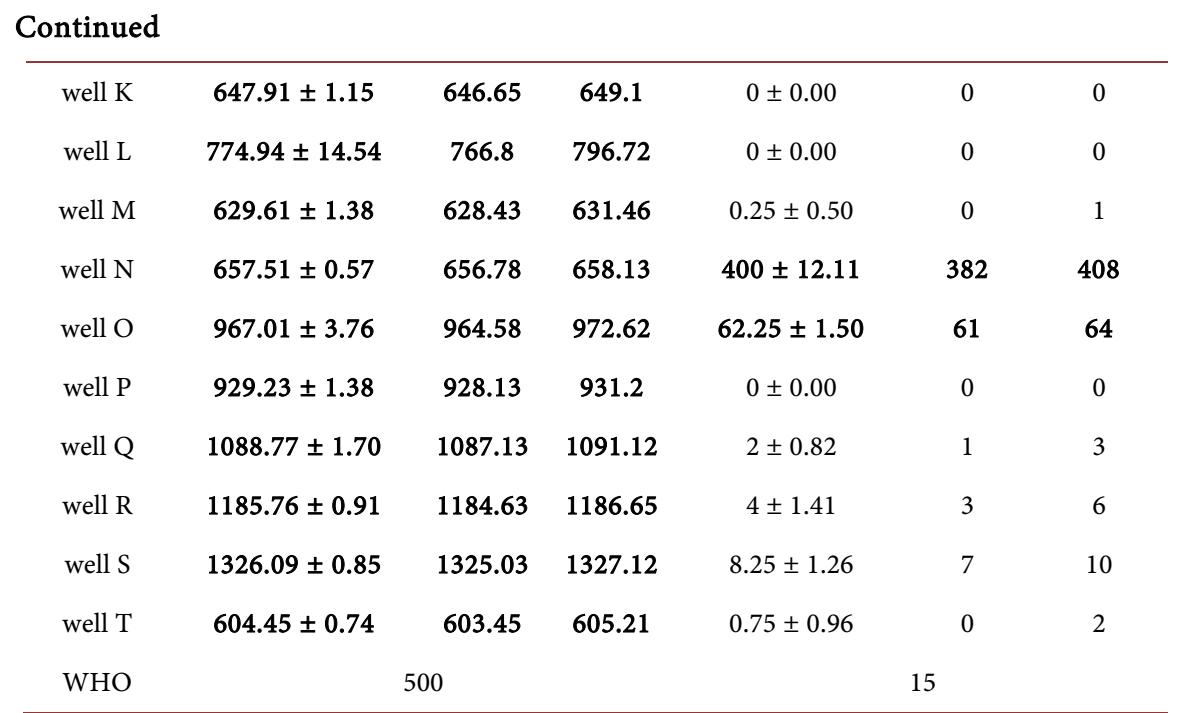

Figures highlighted bold are above the standards.

Table 5. Well water showing mean hardness, magnesium and calcium concentrations.

\begin{tabular}{ccccccc}
\hline \multirow{2}{*}{ WELLS } & \multicolumn{2}{c}{ TOTAL HARDNESS } & & \multicolumn{3}{c}{ ALKALINITY } \\
\cline { 2 - 7 } & MEAN (mg/L) & MIN & MAX & MEAN (mg/L) & MIN & MAX \\
\hline well A & $247.75 \pm 2.36$ & 246 & 251 & $271 \pm 4.55$ & 265 & 275 \\
well B & $152 \pm 1.41$ & 150 & 153 & $119.5 \pm 4.20$ & 115 & 125 \\
well C & $188.5 \pm 2.08$ & 186 & 191 & $161.5 \pm 2.38$ & 160 & 165 \\
well D & $254.75 \pm 2.22$ & 252 & 257 & $294.25 \pm 4.35$ & 290 & 300 \\
well E & $321.5 \pm 1.73$ & 320 & 324 & $226 \pm 6.38$ & 220 & 235 \\
well F & $360.5 \pm 3.32$ & 356 & 363 & $208.75 \pm 6.29$ & 200 & 215 \\
well G & $474.75 \pm 2.22$ & 472 & 477 & $410.5 \pm 5.26$ & 405 & 415 \\
well H & $192.5 \pm 1.91$ & 190 & 194 & $203 \pm 2.45$ & 200 & 205 \\
well I & $46.85 \pm 0.45$ & 46.3 & 47.4 & $75.5 \pm 6.66$ & 70 & 85 \\
well J & $297.85 \pm 1.40$ & 296 & 299.4 & $189 \pm 4.55$ & 185 & 195 \\
well K & $129.95 \pm 1.00$ & 129.2 & 131.4 & $16.75 \pm 2.36$ & 15 & 20 \\
well L & $150.25 \pm 1.71$ & 148 & 152 & $11.5 \pm 2.38$ & 10 & 15 \\
well M & $106.5 \pm 0.58$ & 106 & 107 & $20 \pm 4.08$ & 15 & 25 \\
well N & $56.45 \pm 1.73$ & 54.8 & 58.4 & $21.75 \pm 2.36$ & 20 & 25 \\
well O & $213.375 \pm 1.01$ & 212.4 & 214.3 & $116.5 \pm 2.38$ & 115 & 120 \\
well P & $215.75 \pm 9.74$ & 209 & 230 & $90.5 \pm 4.20$ & 85 & 95 \\
well Q & $254.75 \pm 6.90$ & 250 & 265 & $21.5 \pm 2.38$ & 20 & 25 \\
well R & $279 \pm 2.45$ & 276 & 281 & $83.5 \pm 2.38$ & 80 & 85 \\
well S & $235.75 \pm 1.26$ & 234 & 237 & $91.75 \pm 2.36$ & 90 & 95 \\
well T & $103.75 \pm 1.26$ & 102 & 105 & $30.25 \pm 4.11$ & 25 & 35 \\
WHO & & 500 & & & 200 & \\
\hline
\end{tabular}

Figures highlighted bold are above the standards. 
Table 6. Well water showing mean magnesium and calcium concentrations.

\begin{tabular}{|c|c|c|c|c|c|c|}
\hline \multirow{2}{*}{ WELLS } & \multicolumn{3}{|c|}{ MAGNESIUM } & \multicolumn{3}{|c|}{ CALCIUM } \\
\hline & MEAN (mg/L) & MIN & MAX & MEAN (mg/L) & MIN & MAX \\
\hline well A & $22.343 \pm 0.62$ & 23.144 & 21.831 & $63.01 \pm 28.23$ & 65.73 & 61.34 \\
\hline well B & $16.268 \pm 0.34$ & 16.632 & 15.874 & $33.54 \pm 15.01$ & 34.10 & 32.41 \\
\hline well C & $19.441 \pm 0.12$ & 19.536 & 19.264 & $43.40 \pm 19.53$ & 45.47 & 40.38 \\
\hline well D & $27.435 \pm 1.62$ & 28.882 & 25.123 & $56.48 \pm 25.27$ & 57.24 & 55.47 \\
\hline well E & $25.385 \pm 0.42$ & 26.005 & 25.125 & $86.66 \pm 38.78$ & 87.97 & 84.54 \\
\hline well F & $34.475 \pm 0.39$ & 35.031 & 34.212 & $87.64 \pm 39.20$ & 88.30 & 86.99 \\
\hline well G & $45.036 \pm 2.81$ & 48.621 & 41.742 & $114.45 \pm 51.72$ & 120.10 & 101.98 \\
\hline well $\mathrm{H}$ & $26.289 \pm 0.65$ & 26.991 & 25.421 & $34.06 \pm 15.23$ & 34.64 & 33.66 \\
\hline well I & $1.266 \pm 0.26$ & 1.602 & 1.061 & $16.81 \pm 7.52$ & 17.15 & 16.57 \\
\hline well J & $29.237 \pm 0.98$ & 30.102 & 27.901 & $71.73 \pm 32.10$ & 73.10 & 70.10 \\
\hline well $\mathrm{K}$ & $7.234 \pm 1.10$ & 8.21 & 6.022 & $40.10 \pm 17.93$ & 40.33 & 40.00 \\
\hline well L & $11.595 \pm 0.82$ & 12.712 & 10.731 & $40.62 \pm 18.19$ & 41.81 & 39.02 \\
\hline well M & $8.476 \pm 1.26$ & 9.734 & 6.934 & $29.41 \pm 13.17$ & 30.34 & 28.68 \\
\hline well $\mathrm{N}$ & $4.517 \pm 0.76$ & 5.352 & 3.741 & $39.66 \pm 46.78$ & 114.64 & 14.35 \\
\hline well O & $14.577 \pm 0.76$ & 15.648 & 13.914 & $61.49 \pm 27.53$ & 63.14 & 60.17 \\
\hline well P & $21.453 \pm 0.24$ & 21.792 & 21.284 & $51.43 \pm 23.00$ & 51.82 & 51.04 \\
\hline well Q & $23.867 \pm 1.43$ & 25.081 & 21.982 & $63.21 \pm 28.27$ & 63.44 & 63.00 \\
\hline well $\mathrm{R}$ & $24.208 \pm 1.20$ & 25.832 & 22.963 & $70.57 \pm 31.56$ & 71.09 & 70.31 \\
\hline well S & $23.717 \pm 1.06$ & 25.036 & 22.541 & $55.49 \pm 24.82$ & 55.91 & 55.15 \\
\hline well $\mathrm{T}$ & $16.073 \pm 0.75$ & 16.835 & 15.053 & $14.47 \pm 6.49$ & 14.99 & 13.78 \\
\hline WHO & & & & & & \\
\hline
\end{tabular}

Figures highlighted bold are above the standards.

Table 7. Well water showing mean iron and manganese concentrations.

\begin{tabular}{ccccccc}
\hline \multirow{2}{*}{ WELLS } & \multicolumn{2}{c}{ IRON } & \multicolumn{3}{c}{ MANGANESE } \\
\cline { 2 - 7 } & MEAN (mg/L) & MIN & MAX & MEAN (mg/L) & MIN & MAX \\
\hline well A & $0.09 \pm 0.01$ & 0.08 & 0.1 & $0.06 \pm 0.00$ & 0.06 & 0.06 \\
well B & $0.07 \pm 0.01$ & 0.06 & 0.09 & $0.12 \pm 0.00$ & 0.11 & 0.12 \\
well C & $0.13 \pm 0.02$ & 0.11 & 0.15 & $0.12 \pm 0.00$ & 0.12 & 0.13 \\
well D & $\mathbf{0 . 6 4} \pm \mathbf{0 . 0 1}$ & 0.63 & 0.65 & $0.35 \pm 0.00$ & 0.35 & 0.36 \\
well E & $0.06 \pm 0.01$ & 0.04 & 0.07 & $0.05 \pm 0.00$ & 0.04 & 0.05 \\
well F & $0.19 \pm 0.02$ & 0.17 & 0.21 & $0.09 \pm 0.00$ & 0.09 & 0.09 \\
well G & $\mathbf{0 . 8 3} \pm \mathbf{0 . 0 2}$ & 0.81 & 0.84 & $0.21 \pm 0.01$ & 0.21 & 0.22 \\
well H & $0.09 \pm 0.01$ & 0.07 & 0.1 & $0.13 \pm 0.06$ & 0.10 & 0.22 \\
well I & $\mathbf{1 . 6 8} \pm \mathbf{0 . 0 1}$ & 1.67 & 1.69 & $0.24 \pm 0.00$ & 0.24 & 0.24 \\
\hline
\end{tabular}




\begin{tabular}{ccccccc} 
Continued & \multicolumn{7}{c}{ ( } & & & & \\
well J & $0.34 \pm 0.01$ & 0.33 & 0.35 & $0.04 \pm 0.00$ & 0.04 & 0.05 \\
well K & $0.07 \pm 0.01$ & 0.06 & 0.08 & $0.02 \pm 0.01$ & 0.01 & 0.02 \\
well L & $0.10 \pm 0.02$ & 0.08 & 0.12 & $0.02 \pm 0.01$ & 0.01 & 0.03 \\
well M & $0.02 \pm 0.01$ & 0.01 & 0.04 & $0.00 \pm 0.00$ & 0.00 & 0.00 \\
well N & $\mathbf{0 . 4 8} \pm 0.03$ & 0.45 & 0.51 & $0.02 \pm 0.01$ & 0.01 & 0.03 \\
well O & $\mathbf{0 . 3 7} \pm 0.06$ & 0.29 & 0.42 & $0.03 \pm 0.01$ & 0.02 & 0.03 \\
well P & $0.07 \pm 0.01$ & 0.05 & 0.07 & $0.03 \pm 0.01$ & 0.03 & 0.04 \\
well Q & $0.19 \pm 0.01$ & 0.17 & 0.19 & $0.02 \pm 0.01$ & 0.01 & 0.03 \\
well R & $0.08 \pm 0.01$ & 0.06 & 0.09 & $0.01 \pm 0.01$ & 0.01 & 0.02 \\
well S & $0.07 \pm 0.01$ & 0.06 & 0.08 & $0.02 \pm 0.00$ & 0.02 & 0.03 \\
well T & $0.15 \pm 0.04$ & 0.1 & 0.2 & $0.00 \pm 0.00$ & 0.00 & 0.00 \\
WHO & & 0.3 & & & 0.4 & \\
\hline
\end{tabular}

Figures highlighted bold are above the standards.

Table 8. Well water showing mean potassium and fluorine concentrations.

\begin{tabular}{|c|c|c|c|c|c|c|}
\hline \multirow{2}{*}{ WELLS } & \multicolumn{3}{|c|}{ POTASSIUM } & \multicolumn{3}{|c|}{ FLUORIDE } \\
\hline & MEAN (mg/L) & MIN & MAX & MEAN (mg/L) & MIN & MAX \\
\hline well A & $51.60 \pm 25.80$ & 51.5 & 51.8 & $0.01 \pm 0.01$ & 0 & 0.03 \\
\hline well B & $96.83 \pm 0.79$ & 96.3 & 98 & $0.40 \pm 0.03$ & 0.37 & 0.43 \\
\hline well C & $56.80 \pm 0.57$ & 56.2 & 57.5 & $0.16 \pm 0.01$ & 0.14 & 0.17 \\
\hline well D & $29.63 \pm 2.06$ & 27 & 31.5 & $0.69 \pm 0.02$ & 0.67 & 0.71 \\
\hline well $\mathrm{E}$ & $40.20 \pm 0.98$ & 39 & 41 & $0.58 \pm 0.02$ & 0.56 & 0.61 \\
\hline well F & $191.05 \pm 1.79$ & 189 & 193 & $0.16 \pm 0.01$ & 0.14 & 0.17 \\
\hline well G & $204.75 \pm 1.39$ & 202.9 & 206 & $0.02 \pm 0.01$ & 0.01 & 0.03 \\
\hline well $\mathrm{H}$ & $11.50 \pm 1.00$ & 10 & 12 & $0.77 \pm 0.02$ & 0.75 & 0.79 \\
\hline well I & $30.83 \pm 1.29$ & 29 & 32 & $0.12 \pm 0.01$ & 0.1 & 0.13 \\
\hline well J & $133.25 \pm 1.19$ & 132 & 134.5 & $0.68 \pm 0.01$ & 0.66 & 0.69 \\
\hline well $\mathrm{K}$ & $90.68 \pm 0.53$ & 90.2 & 91.4 & $0.57 \pm 0.01$ & 0.55 & 0.58 \\
\hline well L & $102.28 \pm 0.62$ & 101.7 & 103.1 & $0.23 \pm 0.01$ & 0.21 & 0.24 \\
\hline well M & $98.83 \pm 0.49$ & 98.2 & 99.3 & $0.83 \pm 0.01$ & 0.82 & 0.84 \\
\hline well $\mathrm{N}$ & $19.80 \pm 0.29$ & 19.5 & 20.2 & $0.42 \pm 0.01$ & 0.41 & 0.43 \\
\hline well O & $67.00 \pm 0.82$ & 66 & 68 & $0.69 \pm 0.00$ & 0.68 & 0.69 \\
\hline well P & $41.60 \pm 0.93$ & 40.5 & 42.6 & $0.58 \pm 0.02$ & 0.57 & 0.61 \\
\hline well Q & $55.75 \pm 1.50$ & 54 & 57 & $1.13 \pm 0.02$ & 1.11 & 1.15 \\
\hline well $\mathrm{R}$ & $42.63 \pm 1.25$ & 41 & 44 & $0.71 \pm 0.01$ & 0.69 & 0.72 \\
\hline well S & $33.08 \pm 0.45$ & 32.5 & 33.6 & $0.93 \pm 0.01$ & 0.91 & 0.94 \\
\hline WHO & \multicolumn{3}{|c|}{30} & \multicolumn{3}{|c|}{1.5} \\
\hline
\end{tabular}

Figures highlighted bold are above the standards. 
Table 9. Well water showing mean phosphate and microbial concentrations.

\begin{tabular}{|c|c|c|c|c|c|c|}
\hline \multirow{2}{*}{ WELLS } & \multicolumn{3}{|c|}{ PHOSPHATE } & \multicolumn{3}{|c|}{ MICROBIAL } \\
\hline & MEAN (mg/L) & MIN & MAX & MEAN MPN/100mL & MIN & MAX \\
\hline well A & $4.28 \pm 0.03$ & 4.25 & 4.31 & $37.5 \pm 4.43$ & 33 & 43 \\
\hline well B & $2.84 \pm 0.05$ & 2.8 & 2.92 & $32.5 \pm 0.58$ & 32 & 33 \\
\hline well C & $0.74 \pm 0.39$ & 0.15 & 0.99 & $35 \pm 7.16$ & 27 & 42 \\
\hline well D & $1.47 \pm 0.15$ & 1.25 & 1.57 & $26.75 \pm 4.35$ & 23 & 33 \\
\hline well E & $1.75 \pm 0.07$ & 1.7 & 1.85 & $29.5 \pm 2.08$ & 27 & 32 \\
\hline well F & $3.21 \pm 0.05$ & 3.15 & 3.25 & $10.25 \pm 1.50$ & 8 & 11 \\
\hline well G & $1.99 \pm 0.11$ & 1.9 & 2.15 & $29.25 \pm 3.86$ & 24 & 33 \\
\hline well $\mathrm{H}$ & $0.79 \pm 0.09$ & 0.7 & 0.91 & $21 \pm 6.53$ & 13 & 29 \\
\hline well I & $0.38 \pm 0.03$ & 0.35 & 0.41 & $34 \pm 7.53$ & 28 & 45 \\
\hline well J & $0.39 \pm 0.40$ & 0.17 & 0.98 & $28.25 \pm 6.60$ & 21 & 36 \\
\hline well $\mathrm{K}$ & $0.44 \pm 0.05$ & 0.41 & 0.51 & $5.25 \pm 0.96$ & 4 & 6 \\
\hline well L & $0.07 \pm 0.01$ & 0.05 & 0.08 & $49.5 \pm 4.43$ & 44 & 54 \\
\hline well M & $0.28 \pm 0.09$ & 0.2 & 0.4 & $52.25 \pm 5.38$ & 46 & 59 \\
\hline well $\mathrm{N}$ & $0.23 \pm 0.05$ & 0.2 & 0.3 & $51 \pm 5.60$ & 43 & 56 \\
\hline well O & $0.69 \pm 0.02$ & 0.66 & 0.71 & $43.75 \pm 9.29$ & 31 & 53 \\
\hline well P & $2.06 \pm 0.03$ & 2.02 & 2.1 & $47.75 \pm 9.07$ & 41 & 61 \\
\hline well Q & $3.01 \pm 0.08$ & 2.94 & 3.12 & $44 \pm 7.48$ & 34 & 52 \\
\hline well $\mathrm{R}$ & $1.60 \pm 0.03$ & 1.58 & 1.64 & $49.5 \pm 5.57$ & 42 & 55 \\
\hline well S & $1.80 \pm 0.04$ & 1.75 & 1.84 & $2.75 \pm 0.50$ & 2 & 3 \\
\hline well $\mathrm{T}$ & $0.29 \pm 0.09$ & 0.2 & 0.4 & $53.25 \pm 1.26$ & 52 & 55 \\
\hline WHO & & 5 & & 0 & & \\
\hline
\end{tabular}

Figures highlighted bold are above the standards.

flux level through a sample [13]. It is also a measure of how cloudy water is and may be caused by clays, organic matter, planktons, and other tiny inorganic particles [14]. Turbidity is not a major health concern but high turbidity interferes with disinfection and makes water unattractive and has a high potential to stimulate microbial growth [15]. All the wells besides $\mathrm{N}$ and $\mathrm{O}$ recorded turbidity within the permissible level. The high turbidity recorded in wells $\mathrm{N}$ and $\mathrm{O}$ may be due to the unprotected and shallow nature of the wells and the absence of good lining. The wells, because they are not well protected, have a high potential of allowing organic particles, such as decomposed plant and animal matter, or living biological organisms and inorganic particles (silt, clay, and natural chemical compounds like calcium carbonate) to be washed into the well. There is no standard for total suspended solids (TSS) in drinking water; however, the presence of TSS in drinking water has many implications on the quality of the drinking water source. It can serve as a habitat for microbes to grow as well as a source of attachment for other chemical species. TSS also reduces the effective- 
ness of disinfection in water treatment by shielding microbes. All the wells recorded TSS below $10 \mathrm{mg} / \mathrm{L}$ except well $\mathrm{G}$ which recorded TSS of $14 \mathrm{mg} / \mathrm{L}$.

The measure of conductivity is the waters ability to conduct electric current. It is related to the ionic content of dissolved ions in water. When there are fluctuations in the conductivity of a water source, its safety becomes questionable [16]. Especially in the case of underground water, the conductivity remaining almost the same is an indication that the groundwater is not changing after a period of abstraction or pumping. Chemically pure water must have low conductivity. The conductivity of water ranges from 894 to $3064 \mathrm{mg} / \mathrm{L}$. The mean conductivity of 15 out of the 20 wells was far above the WHO standards (Table 3). Well G which didn't have any protective covering and was also not lined recorded the highest conductivity of $3064 \mathrm{mg} / \mathrm{L}$. A concentration of $500 \mathrm{mg} / \mathrm{L}$ is permitted for TDS in drinking water. All the wells recorded TDS above the allowable concentration except well $\mathrm{J}$ which recorded a minimum value of $127.2 \mathrm{mg} / \mathrm{L}$ during one of the sampling periods (Table 3). One of the major reasons for the high levels of TDS is the non-protective nature of the wells that permits a free flow of runoff into the wells during runoff. The objection to color in water is purely aesthetical but not public health reasons. However, sometimes the presence of color may serve as a source of possible contamination during water treatment. Any observed color in groundwater may be due to certain elements such as iron and manganese in the water or the case of unprotected wells runoffs of silts, clay, decomposed leaves, etc. from the environment. Eight wells out of the 20 recorded color values above the WHO standards (Table 3 ).

Water is said to be hard due to the presence of certain positively charged ions mainly calcium and magnesium dissolved in the water. Other ions such as manganese, iron, strontium, and barium may also cause hardness however, they are usually present in smaller concentrations. Hardness varies greatly due to geological differences [8] and as a result, there are no general standards for hardness. However, total hardness in the range of 0 to $60 \mathrm{mg} / \mathrm{L}$ is said to be soft, those from 61 to $120 \mathrm{mg} / \mathrm{L}$ are moderate, from 120 to $180 \mathrm{mg} / \mathrm{L}$ are hard and above 180 are very hard. The mean total hardness recorded for the wells ranged from 46 to $474 \mathrm{mg} / \mathrm{L}$. Only wells I and $\mathrm{N}$ can be described as soft (Table 4) according to the categorization of hardness above. The wells $\mathrm{T}$ and $\mathrm{M}$ fall under the moderately hard category of water. Three of the wells namely, B, K, and L are hard water whereas the remaining 14 wells measured concentrations in the very hard category. The majority of the wells recording concentrations in the very hard range may be due to seawater intrusion as the wells are close to the coast. Water when very hard though gives objectionable taste has the potential of contributing to the supplementary calcium and magnesium intake [17]. There is an inverse relationship between hard water and hypertension. Drinking soft water increases cardiovascular risk and this effect is mutually reduced by consumption of hard water [1] [18]. Alkalinity is a measure of the buffering capacity of water or the ability of the water to resist change in $\mathrm{pH}$. Groundwater sources with very high alkalinity can resist major changes in pH. Levels of $20-200 \mathrm{mg} / \mathrm{L}$ are typical of 
freshwater and values below $10 \mathrm{mg} / \mathrm{L}$ indicate poor buffering ability. The well $\mathrm{L}$ recorded the minimum mean alkalinity of $11.5 \mathrm{mg} / \mathrm{L}$ whereas well $\mathrm{G}$ recorded the highest mean alkalinity of $410.5 \mathrm{mg} / \mathrm{L}$. The following wells namely: A, D, E, F, G, and $\mathrm{H}$ recorded high alkalinity above the freshwater threshold. This may be a sign of basic compounds being introduced into these wells from runoff due to the lack of well protective covering (Table 3).

A major element like magnesium is essential for the human body in concentrations between $1-10 \mathrm{mg} / \mathrm{L}$ [6]. Magnesium ( $\mathrm{Mg}$ ) and calcium (Ca) are usually introduced into groundwater through the interaction between the water and the solid rocks. Mostly $\mathrm{Ca}$ and $\mathrm{Mg}$ are found in rocks such as limestone, dolomite, and gypsum. $\mathrm{Ca}$ and $\mathrm{Mg}$ in their rightful concentration play a very important role in maintaining a healthy human body. It has been scientifically proven that dietary $\mathrm{Mg}$ is the main source of body $\mathrm{Mg}$ intake however $\mathrm{Mg}$ in water appears hydrated making it more easily absorbed than $\mathrm{Mg}$ in food [8]. The recommended dietary $\mathrm{Mg}$ and $\mathrm{Ca}$ intake is $6 \mathrm{mg} / \mathrm{kg} /$ day and $2500 \mathrm{mg} /$ day respectively [19] [18]. According to [8], the intake of these minerals in the diet is below the recommended value and their presence in water can serve as a supplement. A mean value of $1.2-45 \mathrm{mg} / \mathrm{L}$ and $14.47-114.45 \mathrm{mg} / \mathrm{L}$ were measured for $\mathrm{Mg}$ and $\mathrm{Ca}$ respectively. The levels of $\mathrm{Mg}$ and $\mathrm{Ca}$ measured can supplement their levels in humans however with higher values of 45 and $114 \mathrm{mg} / \mathrm{L}$ of $\mathrm{Mg}$ and $\mathrm{Ca}$ (Table 6), there is a probability that their role as a supplement may be exceeded. The higher concentrations of $\mathrm{Mg}$ and $\mathrm{Ca}$ should be a concern because the major human $\mathrm{Mg}$ and $\mathrm{Ca}$ are usually obtained from diet but not water. Even though low magnesium levels have been implicated in hypertension, higher levels of $\mathrm{Mg}$ in water are also known to have a laxative effect [19]. According to [18] a $1 \mathrm{~kg}$ weight requires $6 \mathrm{mg}$ of $\mathrm{Mg}$ in a day. It, therefore, implies that an $8 \mathrm{~kg}$ weight will require about $48 \mathrm{mg}$ of $\mathrm{Mg}$ in a day. About $55 \%$ of the wells $(20 \mathrm{mg} / \mathrm{L}>)$ are likely to cause laxative problems from their usage especially if people who drink from such well get the average concentration of $507 \mathrm{mg} /$ day $\mathrm{Mg}$ from their diet [8]. Low levels of Ca have been associated with hypertension. Besides high levels of $\mathrm{Ca}$, intake is directed primarily to those who are prone to milk-alkali syndrome and hypercalcemia. This latter condition is not likely to happen in any of the water users as the maximum value of $114 \mathrm{mg} / \mathrm{L}$ would hardly lead to increasing the Ca supplement beyond the maximum of $2500 \mathrm{mg} /$ day [19]. This is because even if the average liters of water an individual consumes under hot weather conditions is $6-10 \mathrm{~L} /$ day, the maximum value of $1140 \mathrm{mg} / \mathrm{L} / \mathrm{day}$ calcium would be consumed. This, when added to the average, $\mathrm{Ca}$ as proposed by Yang and Chiuis not likely to add up to the daily maximum intake $2500 \mathrm{mg} /$ day Ca [8]. Calcium ingested from food and water plays an important role in preventing kidney stones as they bind oxalic acid (a precursor to common kidney stones) and reduce its absorption in the human body [19]. The metal Ca is an essential element for nerve impulse transmission and blood clotting [20].

There are two main sources of iron $(\mathrm{Fe})$ and manganese $(\mathrm{Mn})$ in groundwater, and they are natural and anthropogenic. Naturally, iron and manganese may be 
released into groundwater during the weathering of iron and manganese bearing rocks. Anthropogenically, these minerals may be introduced into groundwater from landfill leachate, industrial and commercial effluent, and acid-mine drainage. The metals $\mathrm{Fe}$ and $\mathrm{Mn}$ are essential minerals which the body requires in small concentrations. The measured concentration of manganese was within the WHO threshold (Table 7). Iron concentration when high in drinking water causes the water to be turbid through the oxidation of its plus two $\left(\mathrm{Fe}^{2+}\right)$ state to the plus three $\left(\mathrm{Fe}^{3+}\right)$ state to form a precipitate. Iron even though has no public health effect in the water, it has the potential to harbor microbial contaminants in drinking water when it precipitates. The mean concentration of iron measured ranged between $0.02-1.68 \mathrm{mg} / \mathrm{L}$ (Table 7). The lowest and highest concentration of iron was recorded in wells $M$ and I respectively. Six of the wells ( $D$, $\mathrm{G}, \mathrm{I}, \mathrm{J}, \mathrm{N}, \mathrm{O}$ ) recorded iron concentrations above the threshold for drinking water. The concentration of iron in the six wells have the potential to change from the plus two $\left(\mathrm{Fe}^{2+}\right)$ to the plus three $\left(\mathrm{Fe}^{3+}\right)$ state and exhibits its consequential effect.

The Recommended Dietary Allowance (RDA) of potassium for adults is 4700 mg per day [21]. Studies have shown that increase exposure to potassium may have significant health effects in people with kidney disease or other conditions, such as coronary artery disease, heart disease, diabetes, hypertension, and adrenal insufficiency [22]. The role of potassium in maintaining normal osmotic pressure in a living cell is a scientific fact. The lowest and highest mean potassium concentration were recorded in wells $\mathrm{H}$ and $\mathrm{G}$ (Table 8). All the wells except $\mathrm{D}, \mathrm{H}$, and $\mathrm{N}$ recorded concentrations within the WHO recommended threshold of $30 \mathrm{mg} / \mathrm{L}$. The rest of the wells recording very high values is very worrying because some of the wells such as G, F, J, and L recorded concentrations which are about 7, 6, 4, and 3 times the threshold concentrations. The public health implications of this later observation are worrying especially in an environment where the sick struggle to get access to doctors: one could just imagine the number of people who will voluntarily go for checkup. People who drink from these water sources may end up with kidney stones without knowing the cause since very little knowledge of high levels of potassium causing kidney stones is known by such groups.

Fluorine usually exists as fluorides in mineral rocks such as cryolite, fluorspar, and fluorapatite. Their level in underground water varies with the type of rock formation the water flows through [22]. Fluoride concentration as high as 8 $\mathrm{mg} / \mathrm{L}$ has been recorded in groundwater in Tanzania. In Ghana, high fluoride levels have been reported in the Bongo district in the northern region of Ghana [23]. Epidemiological studies reveal that long term exposure to fluoride through drinking water has both bones and teeth problems. The mean fluoride concentration measured in the study area was in the range of $0.01-1.13 \mathrm{mg} / \mathrm{L}$ and is within the WHO threshold.

All the wells recorded phosphate levels far below the WHO threshold. None of the wells was found to be microbially safe for drinking purposes (Table 9). The 
results of the water quality index of 9 selected parameters which influence the quality of the groundwater in the area are displayed in Table 10. The parameters selected are those of drinking water concerned in Ghana. The nature of the wells and the commercial and domestic activities which take place in the study area were also considered in the selection. The wells show different levels of quality category. The wells $\mathrm{E}, \mathrm{K}, \mathrm{M}, \mathrm{P}, \mathrm{R}$, and $\mathrm{S}$ were in the range of 0 - 25. This implies that the wells are in an excellent state. The wells A, B, C, F, H, L, Q, and T are in a good category. The well $\mathrm{J}$ and $\mathrm{O}$ are in a poor and very poor state making them unfit for domestic activities. Four of the wells, namely N, I, G, and D had WQI values above 100 making them undesirable for drinking purposes. It must be emphasized here that even though some of the wells were in an excellent and good state, they were however not microbially safe as discussed earlier to be used for drinking purposes. The polluting effect of the individual parameters is presented in Table 11. The table confirms the polluting effect of parameters such as total dissolved solids in all the wells and that of potassium in 17 out of the 20 wells. This latter revelation is an indication that none of the wells is safe for drinking without some form of treatment.

Table 10. Water quality index of the wells.

\begin{tabular}{|c|c|c|c|c|c|c|c|c|c|c|}
\hline $\begin{array}{c}\text { Sample } \\
\text { ID }\end{array}$ & $\begin{array}{c}\mathrm{pH} \\
\mathrm{WnQn}\end{array}$ & $\begin{array}{c}\text { Turb } \\
\text { QnWn }\end{array}$ & $\begin{array}{c}\text { Cond } \\
\text { QnWn }\end{array}$ & $\begin{array}{c}\text { TDS } \\
\text { QnWn }\end{array}$ & $\begin{array}{c}\text { Alk } \\
\text { QnWn }\end{array}$ & $\begin{array}{c}\mathrm{Fe} \\
\text { QnWn }\end{array}$ & $\begin{array}{c}\text { Mn } \\
\text { QnWn }\end{array}$ & $\begin{array}{c}\text { K } \\
\text { QnWn }\end{array}$ & $\begin{array}{c}\mathrm{P} \\
\text { QnWn }\end{array}$ & $\Sigma \mathrm{QnWn}$ \\
\hline well A & 0.048 & 1.459 & & 0.066 & & & & 892 & 2.660 & 26.906 \\
\hline well B & 3.063 & 2.207 & 0.019 & 0.050 & 0.046 & 12.099 & 11.181 & 1.674 & 1.769 & 32.107 \\
\hline well C & 3.219 & 0.840 & 0.018 & 0.049 & 0.063 & 22.901 & 12.104 & 0.982 & 0.457 & 40.634 \\
\hline well D & 1.352 & 1.577 & 0.023 & 0.062 & 0.114 & 110.618 & 34.2 & 0.512 & 0.916 & 149.446 \\
\hline well E & 0.682 & 0.406 & 0.034 & 0.093 & 0.088 & 9.938 & 4.49 & 0.695 & 1.089 & 17.522 \\
\hline well F & 0.766 & 0.288 & 0.035 & 0.095 & 0.081 & 32.408 & 8.677 & 3.302 & 1.994 & 47.646 \\
\hline well G & 1.532 & 2.397 & 0.048 & 0.128 & 0.160 & 143.026 & 20.320 & 3.539 & 1.237 & 172.385 \\
\hline well H & 1.304 & 1.409 & 0.033 & 0.089 & 0.079 & 15.556 & 12.761 & 0.199 & 0.492 & 31.921 \\
\hline well I & 7.347 & 0.425 & 0.014 & 0.038 & 0.029 & 290.805 & 23.236 & 0.533 & 0.236 & 322.663 \\
\hline well J & 1.113 & 1.024 & 0.029 & 0.061 & 0.074 & 58.766 & 4.2 & & 0.240 & 67.814 \\
\hline well $\mathrm{K}$ & 6.366 & 0.862 & 0.015 & 0.040 & 0.007 & 11. & & & & 21.823 \\
\hline well L & 9.345 & 0.784 & 0.018 & 0.048 & 0.004 & 16.420 & & & 0.040 & 30.372 \\
\hline well M & 8.352 & 0.199 & 0.0 & 0.039 & & 3.88 & 0.0 & 1.708 & 0.173 & 14.383 \\
\hline well $\mathrm{N}$ & 7.203 & 31.547 & 0.015 & 0.041 & 0.008 & 82.100 & 1.944 & 0.342 & 0.145 & 123.346 \\
\hline well O & 2.752 & 6.081 & 0.022 & 0.060 & 0.045 & 63.519 & 2.431 & 1.158 & 0.426 & 76.495 \\
\hline well P & 3.721 & 0.376 & 0.021 & 0.058 & 0.035 & 11.235 & 3.160 & 0.719 & 1.279 & 20.604 \\
\hline well Q & 7.311 & 0.314 & 0.025 & 0.068 & 0.008 & 31.976 & 1.944 & 0.964 & 1.870 & 44.480 \\
\hline well R & 0.443 & 0.375 & 0.027 & 0.074 & 0.032 & 13.395 & 1.2 & 0.737 & 0.996 & 17.294 \\
\hline well S & 1.795 & 1.090 & 0.031 & 0.083 & 0.036 & 11.235 & 2.188 & 0.572 & 1.118 & 18.146 \\
\hline well T & 8.412 & 0.904 & 0.014 & 0.038 & 0.012 & 25.494 & 0.000 & 0.819 & 0.177 & 35.869 \\
\hline
\end{tabular}

Figures highlighted bold are above the standards. 
Table 11. Pollution load index of wells.

\begin{tabular}{|c|c|c|c|c|c|c|c|c|c|c|c|c|}
\hline $\begin{array}{c}\text { Sample } \\
\text { ID }\end{array}$ & $\mathrm{pH}$ & Turb & Color & ond & TDS & Alk & Iron & In & $\mathrm{F}$ & K & $\mathrm{P}$ & TH \\
\hline A & 0.930 & 9 & & 2 & 1 & & 0.308 & 56 & 0.008 & 20 & 0.855 & 0.496 \\
\hline well B & 1.022 & 710 & 0 & 1.191 & 1.606 & . & 0.233 & 75 & 0.267 & 3.228 & .569 & 0.304 \\
\hline well C & 1.027 & 0.270 & 0.550 & 1.177 & 1.585 & 0.808 & 0.442 & 3113 & 0.107 & .893 & 0.147 & 0.377 \\
\hline well D & 0.968 & 0.507 & 3 & 1.470 & 1.984 & 1.4 & 2.133 & 8813 & 0.462 & .988 & 0.295 & 0.510 \\
\hline well E & 0 & 0.131 & 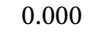 & 2 & 6 & 130 & 92 & 56 & 88 & 40 & .350 & 0.643 \\
\hline well F & 0.950 & 0.093 & 0.833 & 2.257 & 3.039 & 1.044 & 0.625 & 0.2231 & 0.107 & 6.368 & 0.641 & 0.721 \\
\hline well G & 0.973 & 0.771 & 0 & 3.056 & 4.118 & 2.053 & 2.758 & 25 & 0.015 & 25 & 0.398 & 0.950 \\
\hline well $\mathrm{H}$ & 0.966 & 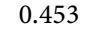 & & & 5 & 1.0 & 0 & & 2 & 3 & 58 & 0.385 \\
\hline well I & 1.189 & 0.137 & 0.000 & 0.897 & 1.211 & 0.378 & 5.608 & 0.5975 & 0.080 & 1.028 & 0.076 & 0.094 \\
\hline $\mathrm{w}$ & 0 & 0 & & 7 & 1 & 0.945 & 3 & & 2 & & 0.077 & c \\
\hline well K & 1.146 & 0.27 & 0.000 & 0.5 & 1.296 & 0.08 & 0. & & 0.3 & & 0.088 & 0.260 \\
\hline well L & 1.288 & 0.252 & 0.000 & 1.137 & 1.550 & 0.058 & 0.317 & 0.0500 & 0.153 & 3.409 & 0.013 & 0.301 \\
\hline we & 1 & 0 & & & & & 0.075 & & & & 56 & \\
\hline well N & 1.183 & 10.140 & 26.667 & 0.975 & 1.315 & 0.109 & 1.583 & 0.0500 & 0.282 & .660 & 0.047 & 0.113 \\
\hline well O & 1.012 & 1.955 & 4.150 & 1.431 & 1.934 & 0.583 & 1.225 & 0.0625 & 0.458 & 2.233 & 0.137 & 0.427 \\
\hline well P & 1.045 & 0 & 0.000 & 1. & 8 & 53 & 0.217 & 0.0813 & 0.387 & 1.387 & 0.411 & 0.432 \\
\hline well Q & 1.188 & 0.101 & 0.133 & 1.617 & 2.178 & 0.108 & 0.617 & 0.0500 & 0.752 & 1.858 & 0.601 & 0.510 \\
\hline well $\mathrm{R}$ & 0.941 & 0.121 & 0.267 & 1.759 & 2.372 & 0.418 & 0.258 & 0.0313 & 0.473 & 1.421 & 0.320 & 0.558 \\
\hline well S & 0.981 & 0.351 & 0.550 & 1.967 & 2.652 & 0.459 & 0.217 & 0.0563 & 0.618 & 1.103 & 0.360 & 0.472 \\
\hline well $\mathrm{T}$ & 240 & 0.291 & 0.050 & 0.897 & 1.209 & 0.151 & 0.492 & 0.0000 & 0.488 & 1.579 & 0.057 & 0.208 \\
\hline
\end{tabular}

Figures highlighted bold are above the standards.

\section{Conclusion}

This study investigated the quality issues associated with wells constructed without following rules and regulations and the potential threat such wells pose to their users. The results of the analysis show that the wells are not safe for drinking and preparation of meals which does not involve boiling before consumption. The wells $\mathrm{E}, \mathrm{K}, \mathrm{M}, \mathrm{P}, \mathrm{R}$, and $\mathrm{S}$ were found to be in excellent condition physicochemically. The wells $\mathrm{A}, \mathrm{B}, \mathrm{C}, \mathrm{F}, \mathrm{H}, \mathrm{L}, \mathrm{Q}$, and $\mathrm{T}$ fell in the good range whereas wells $\mathrm{J}$ and $\mathrm{O}$ are in the poor and very poor category respectively. The remaining wells, namely $\mathrm{N}, \mathrm{I}, \mathrm{G}$, and $\mathrm{D}$ were found to be undesirable for drinking. The pollution load index confirms that none of the wells is safe for drinking. The wells need some level of treatment before they can be used for drinking and other domestic purposes. The high level of TDS also calls for proper protection of the wells to prevent an inflow of runoff since almost all the wells are not well protected. The communities are advised to line and protect the wells to reduce external contamination. 


\section{Acknowledgements}

The authors would like to thank the technicians and staff of Department of Water and Sanitation, University of Cape Coast (UCC) for providing laboratory space, facilities and assistance to support the study. We also express our sincere gratitude to the volunteers from the Abura community who helped by assisting us in the identification of the wells.

\section{Conflicts of Interest}

The authors declare no conflicts of interest regarding the publication of this paper.

\section{References}

[1] Sengupta, P. (2013) Potential Health Impacts of Hard Water. International Journal of Preventive Medicine, 4, 866.

[2] Margat, J. and Van der Gun, J. (2013) Groundwater around the World: A Geographic Synopsis. CRC Press, Boca Raton. https://doi.org/10.1201/b13977

[3] Zektser, I.S. and Everett, L.G. (2004) Groundwater Resources of the World and Their Use.

[4] Siebert, S., Burke, J., Faures, J.M., Frenken, K., Hoogeveen, J., Döll, P. and Portmann, F.T. (2010) Groundwater Use for Irrigation-A Global Inventory. Hydrology and Earth System Sciences, 14, 1863-1880. https://doi.org/10.5194/hess-14-1863-2010

[5] Smith, M., Cross, K., Paden, M. and Laban, P. (2016) Spring-Managing Groundwater Sustainably. IUCN, Gland. https://doi.org/10.2305/IUCN.CH.2016.WANI.8.en

[6] Foster, S., Kemper, K., Tuinhof, A., Koundouri, P., Nanni, M. and Garduno, H. (2006) Natural Groundwater Quality Hazards, Avoiding Problems, and Formulating Mitigation Strategies. GW. MATE Core Group. Briefing Note Series Note, 14.

[7] Raghunath, H.M. (1982) Groundwater-Hydrogeology. Ground Water Survey, and Pumping Tests, Rural Water Supply and Irrigation Systems.

[8] Yang, C.Y. and Chiu, H.F. (1999) Calcium and Magnesium in Drinking Water and the Risk of Death from Hypertension. American Journal of Hypertension, 12, 894-899. https://doi.org/10.1016/S0895-7061(99)00065-5

[9] Linsley, R.K. and Franzini, J.B. (1979) Water Resources Engineering (Vol. 165). McGraw Hill, New York.

[10] Sililo, O.T., Saayman, I.C. and Fey, M.V. (2001) Groundwater Vulnerability to Pollution in Urban Catchments. No. 1008/1/01, Water Research Commission, WRC, Pretoria.

[11] Gale, I.N. and Robins, N.S. (1989) The Sampling and Monitoring of Groundwater Quality. British Geological Survey. Hydrogeology Report, (89/37).

[12] Carr, G.M. and Neary, J.P. (2008) Water Quality for Ecosystem and Human Health. UNEP/Earthprint.

[13] APHA/AWWA/WEF (2012) Standard Method 2130: Turbidity. Standard Methods for the Examination of Water and Wastewater. 22nd Edition, American Public Health Association, American Water Works Association, and Water Environment Federation, Washington DC.

[14] Reckhow, D.A., Linden, K.G., Kim, J., Shemer, H. and Makdissy, G. (2010) Effect of 
UV Treatment on DBP Formation. Journal American Water Works Association, 102, 100-113. https://doi.org/10.1002/j.1551-8833.2010.tb10134.x

[15] World Health Organization (2004) Fluoride in Drinking-Water: Background Document for Development of WHO Guidelines for Drinking-Water Quality.

[16] Duncan, A.E., de Vries, N. and Nyarko, K.B. (2018) Assessment of Heavy Metal Pollution in the Main Pra River and Its Tributaries in the Pra Basin of Ghana. Environmental Nanotechnology, Monitoring \& Management, 10, 264-271. https://doi.org/10.1002/j.1551-8833.2010.tb10134.x

[17] Galan, P., Arnaud, M.J., Czernichow, S., Delabroise, A.M., Preziosi, P., Bertrais, S., Hercberg, S., et al. (2002) Contribution of Mineral Waters to Dietary Calcium and Magnesium Intake in a French Adult Population. Journal of the American Dietetic Association, 102, 1658-1662. https://doi.org/10.1016/S0002-8223(02)90353-6

[18] Durlach, J., Bara, M. and Guiet-Bara, A. (1985) Magnesium Level in Drinking Water and Cardiovascular Risk Factor: A Hypothesis. Magnesium, 4, 5-15. https://doi.org/10.1016/S0750-7658(85)80119-2

[19] Cotruvo, J.A. and Bartram, J. (2009) Calcium and Magnesium in Drinking-Water: Public Health Significance. World Health Organization, Geneva.

[20] Rosborg, I., Nihlgård, B. and Ferrante, M. (2015) Mineral Composition of Drinking Water and Daily Uptake. In: Drinking Water Minerals and Mineral Balance, Springer, Cham, 25-31. https://doi.org/10.1007/978-3-319-09593-6 2

[21] Pohl, H.R., Wheeler, J.S. and Murray, H.E. (2013) Sodium and Potassium in Health and Disease. In: Interrelations between Essential Metal Ions and Human Diseases, Springer, Dordrecht, 29-47. https://doi.org/10.1007/978-94-007-7500-8 2

[22] World Health Organization (2009) Potassium in Drinking-Water: Background Document for Development of WHO Guidelines for Drinking-Water Quality (No. WHO/HSE/WSH/09.01/7). World Health Organization, Geneva.

[23] Firempong, C.K., Nsiah, K., Awunyo-Vitor, D. and Dongsogo, J. (2013) Soluble Fluoride Levels in Drinking Water-A Major Risk Factor of Dental Fluorosis among Children in the Bongo Community of Ghana. Ghana Medical Journal, 47, 16-23. 